\title{
Endometrial Stromal Sarcomas: A Retrospective Analysis of 28 Patients, Single Center Experience for 20 Years
}

\author{
Eun Ji Nam, M.D. ${ }^{1}$, Jae Wook Kim, M.D. ${ }^{2}$, Dae Woo Lee, M.D. ${ }^{1}$, Si Young Jang, M.D. ${ }^{1}$, Jong Wook Hong, \\ M.D. ${ }^{1}$, Young Tae Kim, M.D. ${ }^{1}$, Jae Hoon Kim, M.D. ${ }^{1}$, Sunghoon Kim, M.D. ${ }^{1}$ and Sang Wun Kim, M.D. ${ }^{1}$ \\ ${ }^{1}$ Women's Cancer Clinic, Department of Obstetrics and Gynecology, Yonsei University College of Medicine, Seoul, ${ }^{2}$ Division \\ of Gynecologic Oncology, Department of Obstetrics and Gynecology, Kwandong University College of Medicine, Goyang, \\ Korea
}

Purpose: The aim of this study was to evaluate the behavior of endometrial stromal sarcomas (ESSs) in relation to their clinical and pathogenic features, and to determine the optimal treatment strategy.

Materials and Methods: A retrospective analysis was performed involving 28 patients with histologic-proven ESSs treated at our institution between 1987 and 2006.

Results: The median follow-up was $54.7 \pm 63.1$ months and the 5-year survival rate was $82.0 \%$. Twenty-two $(81.5 \%)$ and 5 patients $(18.5 \%)$ had low- and high-grade disease, respectively. Univariate analysis revealed that the histologic grades, based on mitotic count, were associated with longer survival $(p=0.004)$. However, among those patients with low-grade tumors, 5/20 patients $(25 \%)$ had a recurrence and $2 / 21$ patients $(9.5 \%)$ had distant metastasis during the follow-up period. With the exception of 2 patients, 26 patients with ESSs underwent

\section{INTRODUCTION}

Endometrial stromal sarcomas (ESSs) are very rare, malignant uterine tumors, comprising $<1 \%$ of all uterine cancers (1). Frequently, ESSs are detected by chance at the time of diagnostic dilatation and curettage (D\&C), myomectomy, or hysterectomy for presumed benign uterine disease. Because of the non-specific characteristics of ESSs, little is known regarding the pathogenesis, risk factors, optimal therapy, or outcomes.

The following characteristics have been shown to have prognostic significance for ESSs: tumor size, FIGO stage, grade, age, and menopausal status (2). The most reliable known prognostic factors are the grade of tumor and the extent of disease (3). ESSs can be divided into low- and high-grade ESSs

Correspondence: Jae Wook Kim, Division of Gynecologic Oncology, Department of Obstetrics and Gynecology, Kwandong University College of Medicine, 697-24, Hwajeong-dong, Deokyang-gu, Goyang 412-270, Korea. (Tel) 82-31-810-5003, (Fax) 82-31-964-6649, (E-mail) jwkim0630@kwandong.ac.kr

Received November 16, 2007, Accepted January 28, 2008 hysterectomy as primary treatment. Adjuvant treatment after surgery was administered to $14 / 26$ patients $(53.8 \%)$. Hormone therapy with progesterone, chemotherapy, and/or radiotherapy did not influence overall survival. However, the postoperative adjuvant therapy group, regardless of the treatment modality, was associated with relatively increased overall survival when compared to the surgery only group $(p=0.054)$.

Conclusion: The preoperative differential diagnosis of ESSs from other benign gynecologic diseases is often difficult. We recommend adjuvant therapy be administered after hysterectomy in patients with ESS to prevent recurrence or distant metastasis. (Cancer Res Treat. 2008;40:6-10)

Key Words: Endometrial, Stromal, Sarcoma, Radiotherapy, Chemotherapy, Treatment outcome

based on a mitotic count of less or more than 10 mitoses/10 high power fields and characterized by a proliferation cells with endometrial stromal differentiation (4). Low-grade ESS has an indolent clinical course, whereas high-grade ESS behaves in a more aggressive manner with a poorer overall prognosis. Accordingly, it has been suggested that high-grade ESS should be regarded as a different disease entity altogether (5). However, some authors are of the opinion that mitotic count is not indicative of tumor recurrence or tumor-related deaths (6).

The fundamental treatment for ESSs consists of total hysterectomy with bilateral salpingo-oophorectomy. However, $\mathrm{Li}$ et al(7) recently demonstrated that ovarian preservation could be a safe option for surgical treatment in stage I, low-grade ESS. The importance of surgical staging, including lymphadenectomy, is still unknown (3). Due to the high recurrence risk, even with localized tumors, many clinicians advocate the use of adjuvant therapy (2); however, no prospective studies have been conducted regarding the merits of adjuvant radiation and/or chemotherapy or hormonal treatment. Of note, the overall survival does not appear to improve with adjuvant therapy $(8,9)$.

The aim of this study was to evaluate the behavior of ESSs in relation to its clinical and pathogenic features and to determine the optimal treatment strategy. 


\section{MATERIALS AND METHODS}

This study included 28 patients with histologic-proven ESS treated between 1987 and 2006 in the Department of Obstetrics and Gynecology of Yonsei University College of Medicine. Data were retrospectively reviewed and included age at the time of diagnosis, patient demographics, clinical presentation, extent of surgery performed, adjuvant therapy, patient outcome, recurrence patterns, and follow-up status.

Twenty-two cases of low-grade ESS and 5 cases of highgrade ESS were identified. One case was not classified as either low- or high-grade ESS due to diagnostic ambiguity. Surgical staging was based retrospectively on the 1988 FIGO guidelines for cancer of the uterine corpus (10). Total hysterectomy and removal of as much tumor as possible was performed as a surgical procedures. With respect to the patients who made up the current study, the decision to perform adjuvant therapy was left to the individual physician, thus the adjuvant and non-adjuvant groups were not necessarily similar. The adjuvant group was administered chemotherapy, radiotherapy, or hormonal therapy. Some patients had a combination of the therapies. Megestrol acetate $(120 \sim 320 \mathrm{mg} /$ day for $6 \sim 12$ months) was used as hormonal therapy. Chemotherapeutic regimens were variable; however, mainly consisted of a combination of ifosfamide, adriamycin, and platinum based-agents.

Survival analysis was used to compare patients with or without specific clinicopathologic parameters in terms of progression-free and overall survival. The Kaplan-Meier method was used to estimate survival curves, and the log-rank statistic was used to test the equality of the survival functions between the patients with or without specific clinicopathologic parameters.
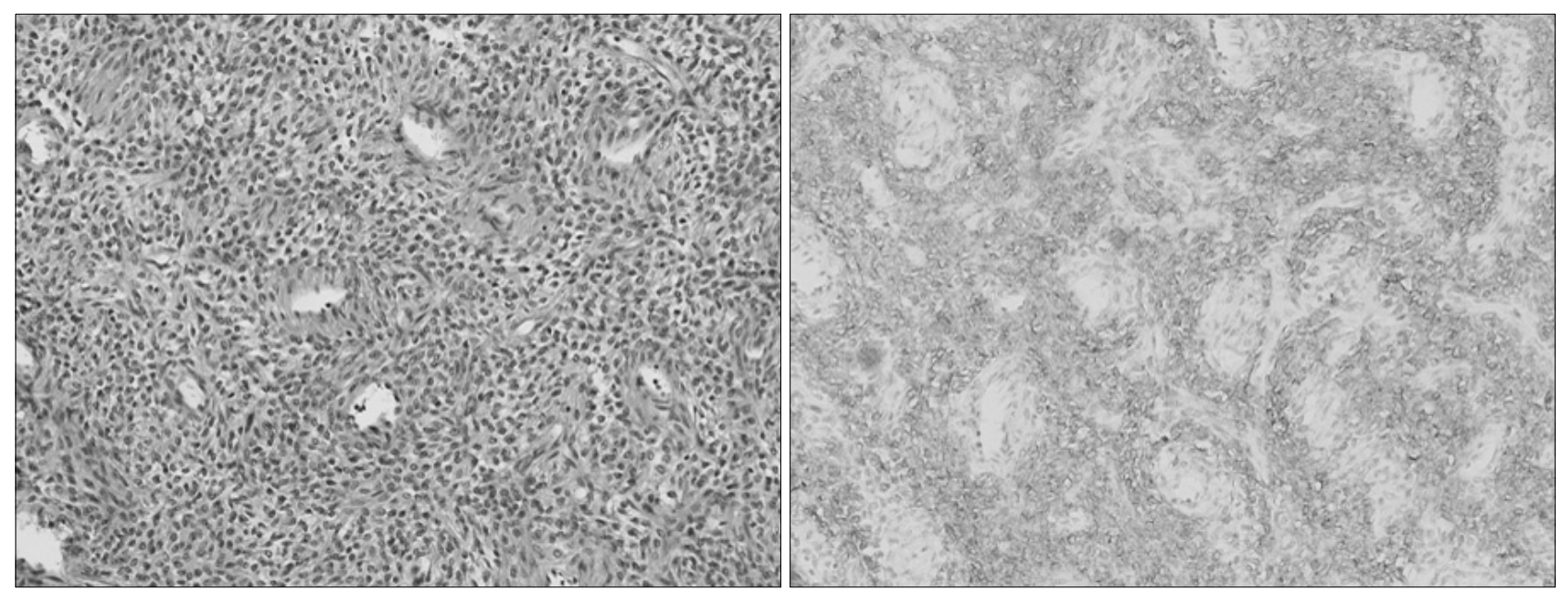

Fig. 1. Low-grade ESS. The tumor resembles normal stroma of proliferative endometrium, characterized by generally uniform cells with minimal nuclear pleomorphism and cytologic atypia, hyalinized connective tissue, and a rich, vascularized background (left, hematoxylin and eosin; right, corresponding part of the left slide stained positive for CD10).
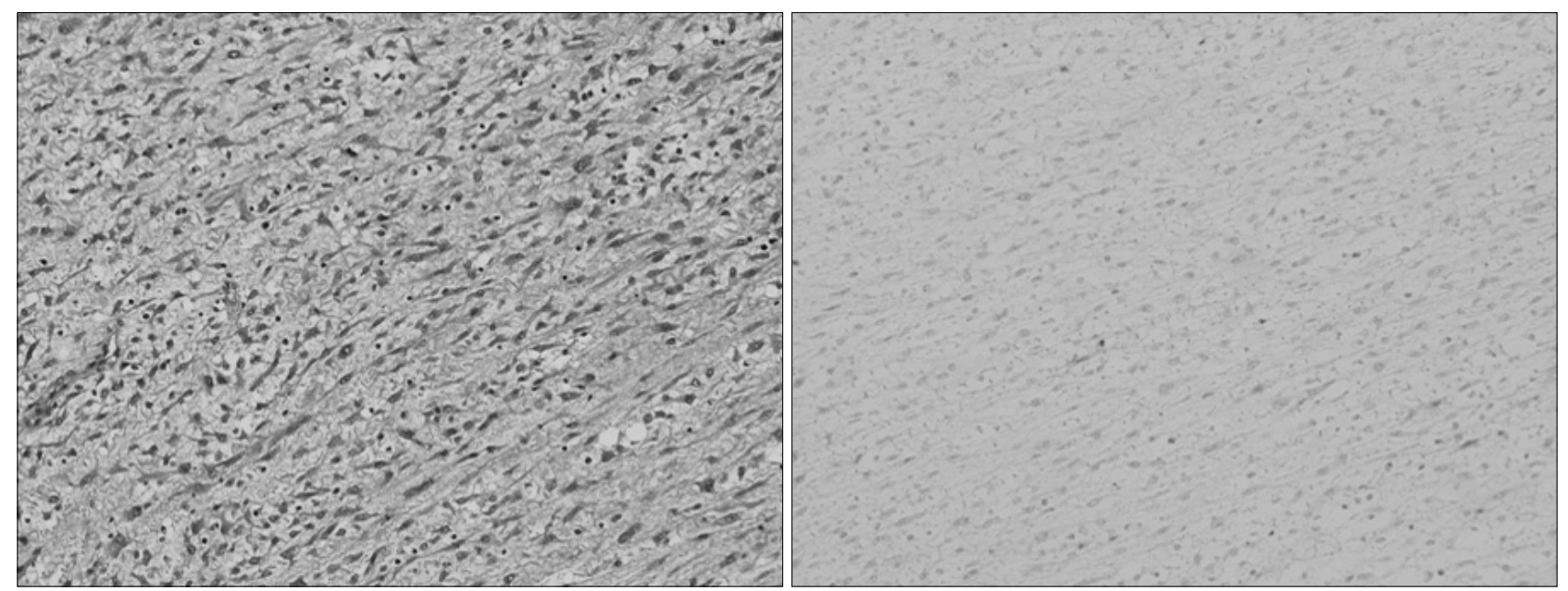

Fig. 2. High-grade ESS. The tumor is composed of anaplastic spindle cells that have a high mitotic index and frequently encountered abnormal mitotic figures. (left, hematoxylin and eosin; right, corresponding part of the left slide stained negative for CD10). 


\section{RESULTS}

The median age at the time of diagnosis was 47.0 years (range, $20 \sim 76$ years). Sixteen patients $(57.1 \%)$ were referred to our institution from private clinics for definitive treatment of ESSs. They had previously undergone either hysterectomy, myomectomy, D\&C, or hysteroscopy for presumed benign gynecologic conditions. Experienced pathologists analyzed all specimens (Fig. 1, 2). Patient characteristics are listed in Table 1.

Twenty-six of 28 patients had undergone total hysterectomy with or without bilateral salpingo-oophorectomy as part of their initial surgical treatment. Two patients had radiotherapy for

Table 1. Patient characteristics

\begin{tabular}{llc}
\hline & Characteristics & Patients $(\mathrm{n}=28)$ \\
\hline Age & Median & 47 \\
& Range & $20 \sim 76$ \\
Stage & I & $16(57.1 \%)$ \\
& III & $4(14.3 \%)$ \\
& IV & $3(10.7 \%)$ \\
Grade & Unknown & $5(17.9 \%)$ \\
& Low & $22(78.6 \%)$ \\
Distant metastasis & High & $5(17.9 \%)$ \\
& Unknown & $1(3.6 \%)$ \\
& No & $22(78.6 \%)$ \\
Hysterectomy & Yes & $5(17.9 \%)$ \\
& Unknown & $1(3.6 \%)$ \\
& Yes & $26(92.9 \%)$ \\
& No & $2(7.1 \%)$ \\
\hline
\end{tabular}

Table 2. Adjuvant therapy after surgical treatment $(n=26)$

\begin{tabular}{lcc}
\hline & $\begin{array}{c}\text { Low-grade ESS } \\
(\mathrm{n}=21)\end{array}$ & $\begin{array}{c}\text { High-grade ESS } \\
(\mathrm{n}=5)\end{array}$ \\
\hline Observation & $9(42.9 \%)$ & $3(60.0 \%)$ \\
$\mathrm{RTx}^{*}$ & $2(9.5 \%)$ & 0 \\
$\mathrm{CTx}^{\dagger}$ & $3(14.3 \%)$ & 0 \\
$\mathrm{HTx}^{\dagger}$ & $5(23.8 \%)$ & 0 \\
$\mathrm{RTx}^{\dagger}+\mathrm{CTx}$ & $1(4.8 \%)$ & $1(20.0 \%)$ \\
$\mathrm{RTx}+\mathrm{HTx}$ & $1(4.8 \%)$ & 0 \\
$\mathrm{RTx}+\mathrm{CTx}+\mathrm{HTx}$ & 0 & $1(20.0 \%)$ \\
\hline
\end{tabular}

*radiotherapy, ${ }^{\dagger}$ chemotherapy, ${ }^{\dagger}$ hormonal treatment.

Table 3. Clinical outcomes of patients

\begin{tabular}{lcc}
\hline \multicolumn{1}{c}{ Outcomes } & $\begin{array}{c}\text { Low-grade ESS } \\
(\mathrm{n}=22)\end{array}$ & $\begin{array}{c}\text { High-grade ESS } \\
(\mathrm{n}=5)\end{array}$ \\
\hline No evidence of disease & $15(68.1 \%)$ & $1(20.0 \%)$ \\
Alive with disease & $4(18.2 \%)$ & 0 \\
Dead of disease & $1(4.5 \%)$ & $2(40.0 \%)$ \\
Missing & $2(9.1 \%)$ & $2(40.0 \%)$ \\
\hline
\end{tabular}

their initial treatment. More than one-half of the patients $(16 / 26$ [61.5\%]) were stage I. Distant metastasis outside the pelvis at the time of diagnosis had occurred in 5 patients (17.9\%); para-aortic lymph node metastasis occurred in 1 patient each from the low- and high-grade ESS. Also, one patient with a high-grade ESS had metastasis to the omentum, and 2 patients with low-grade ESSs had metastasis to the bladder and lung (i.e., stage IV), respectively.

Adjuvant therapy after surgery was performed in 14/26 cases (53.8\%; Table 2). The most common choice for adjuvant therapy in low-grade ESSs was hormonal treatment with megestrol acetate. In 5 patients with high-grade ESSs, 2 patients were treated with radiation therapy in combination with chemotherapy as adjuvant treatment. One of the patients also had hormonal treatment with megestrol acetate $(480 \mathrm{mg} /$ day $)$. One patient with a high-grade ESS voluntarily discontinued adjuvant treatment because of her advanced age, and the other 2 patients voluntarily discontinued treatment due to poor performance status with extensive disease.

The median overall survival was 56.5 months [95\% CI: 47.0 $\sim 97.6]$ and the 5-year survival rate was $82.0 \%$. Five patients

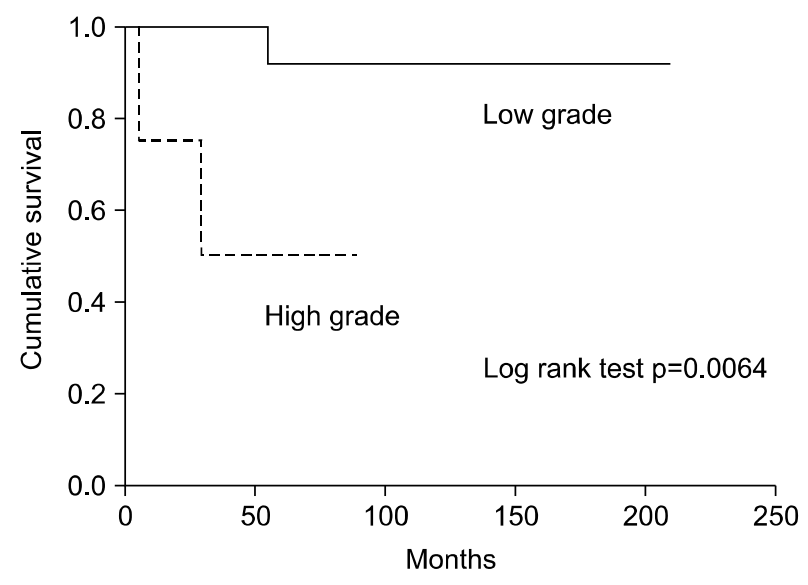

Fig. 3. Overall survival as a function of ESS grade.

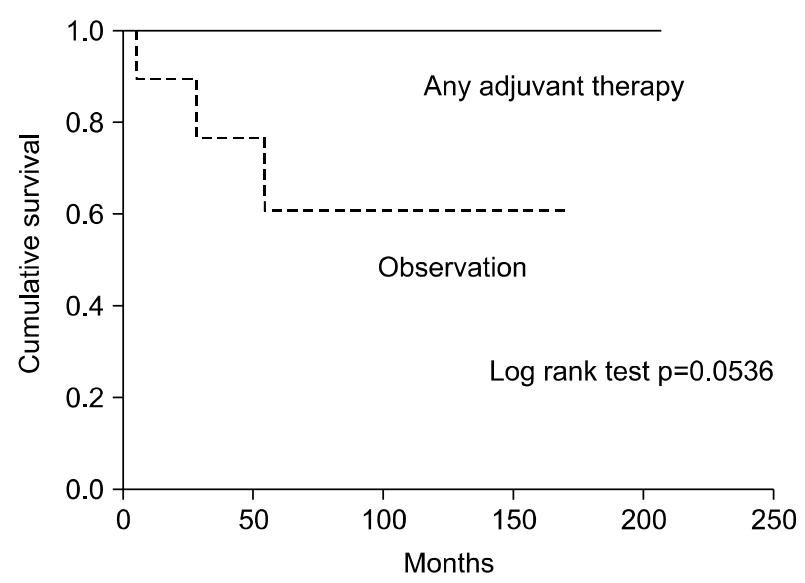

Fig. 4. Overall survival based on adjuvant therapy after surgical treatment. 
(22.7\%) with low-grade ESSs developed disease recurrence, one of whom died from the disease (Table 3). However, only 1 patient (20.0\%) with a high-grade ESS showed no evidence of disease in a recent follow-up visit. Three patients with recurrent, low-grade ESS had intra-abdominal recurrent disease a few years after the initial diagnosis of their disease, while 1 patient had lung metastasis 13 years after diagnosis; the other patient had metastasis to the abdominal wall 12 years after the initial diagnosis.

Univariate analysis revealed that the histologic grade according to the mitotic count was associated with longer survival ( $\mathrm{p}=0.004$; Fig. 3). Other potential prognostic factors, such as stage, existence of lymph node metastasis, and age did not influence either disease-free survival or overall survival. Postoperative adjuvant therapy, hormone therapy, chemotherapy, and/or radiotherapy did not individually influence overall survival. However, the postoperative adjuvant therapy group, regardless of the treatment modality, was associated with relatively increased overall survival when compared to the no adjuvant therapy group ( $p=0.054$; Fig. 4).

\section{DISCUSSION}

ESS is a tumor arising from the cells of the endometrial stroma. It can originate from the endometrium, adenomyosis, and occasionally, endometriosis. Because of the rarity of ESS, only small numbers of retrospective studies exist regarding the early diagnosis, prognostic factors, tumor behavior, and treatment guidelines.

Due to the great similarity of ESS with normal endometrium, it may be impossible to diagnose low-grade ESS with certainty on curettage fragments, and the definitive diagnosis can be made only on a hysterectomy specimen (11). Only 5 patients were diagnosed with malignancy in our series by $\mathrm{D} \& \mathrm{C}$. Imaging studies, such as magnetic resonance imaging or sonography, have only a limited role in the diagnosis of ESS from endometrial cancer, uterine myoma, or adenomyosis $(12,13)$. In the current study, 16/28 (57.1\%) patients were referred from local clinics after hysterectomy, myomectomy, $\mathrm{D} \& \mathrm{C}$, or hysteroscopy for presumed benign gynecologic conditions $(57.1 \%)$. A recent study reported that optimal cytoreductive surgery could improve the median survival of patients with ESSs (14). Appropriate diagnostic modalities would therefore lead to a reduction in the rate of re-operations.

Optimized therapeutic guidelines have not been agreed upon. Surgery has always been described as the most effective treatment for uterine sarcomas $(2,15)$. However, the extent of surgery, especially the role of lymphadenectomy, is controversial. When patients are referred to our institute after hysterectomy, they usually undergo radiation therapy as a primary treatment, whereas when referred after D\&C or myomectomy, patients typically undergo surgical staging, including lymphadenectomy. One study patient who underwent lymphadectomy for low-grade ESS had lymph node metastasis in the para-aortic area. In agreement with our study, recent data suggests that the rate of lymph node involvement in low-grade ESSs may be higher than expected (16). However, the therapeutic benefit of radical lymphadenectomy is still uncertain.

Consistent with previous reports, the grade of tumor was the most significant prognostic factor in our study. Patients with low-grade ESSs had better survival compared to those patients with high-grade ESSs $(3,14,17)$. However, patients with low-grade ESSs are frequently diagnosed in an early tumor stage, but still experience late recurrence or metastasis. Five patients (22.7\%) with low-grade ESSs developed disease recurrence, one of whom died of disease in our series. Therefore, this could be the rationale for adjuvant therapy to prevent late recurrence or metastasis.

Adjuvant therapy for patients with ESSs is still controversial (17). One study reported no difference in the recurrence rate in patients who had surgery with adjuvant therapy versus surgery alone (8). However, with respect to the presumed need to treat those patients perceived to be at higher risk with adjuvant therapy, the current study showed that the postoperative adjuvant therapy group regardless of the treatment modality was associated with relatively increased overall survival when compared to no adjuvant therapy group ( $\mathrm{p}=$ 0.054). Therefore, strong consideration should be given to performing adjuvant therapy, even in patients with low-grade ESSs. Unfortunately, the optimal choice of adjuvant therapy for ESSs is unknown, with options including radiation therapy, chemotherapy, and hormonal therapy either alone or in varying combinations.

Chemotherapeutic regimens utilized to treat ESS have included both single and combination agent therapy, including ifosfamide, cyclophosphamide, doxorubicin, adriamycin, cisplatin, etoposide, parclitaxel, and carboplatin $(15,18 \sim 21)$. Nonrandomized studies have described a favorable response with doxorubicin-based chemotherapy (8). Randomized controlled trials have suggested that the most effective single agent appears to be ifosfamide, with a $33 \%$ response rate (a 14\% complete response and a $19 \%$ partial response) in a phase II trial by the Gynecologic Oncology Group for recurrent or metastatic disease (18)

Similar to chemotherapy, no definite recommendations exist for adjuvant hormone therapy in patients, especially with low-grade ESS. Several reports have been published on the efficacy of progestin $(4,22)$ and aromatase inhibitors in the treatment of metastatic ESS (23). One advantage of adjuvant hormone therapy is that it allows long-term maintenance therapy. In addition, it appears that women with advanced stage disease benefit from adjuvant hormone therapy (24).

A combination of surgery and adjuvant radiotherapy may improve the treatment outcome compared to surgery alone (25). However, Gadducci et al. showed no big difference in the outcome of patients with low-grade ESSs treated by surgery alone and surgery with adjuvant radiotherapy (3). For more convincing results, prospective studies with larger case series are necessary to help define the optimal choice of treatment.

Taken together, we have shown that clinical outcomes can be readily depicted based on the grade of the ESS. Also, we recommend adjuvant therapy to be performed after hysterectomy to prevent recurrence or distant metastasis, even in lowgrade ESSs. 


\section{CONCLUSION}

The differential diagnosis of ESSs from other benign gynecologic diseases before surgery is often difficult. Though it is known that grade is the most important prognostic factor for patients with ESSs, recurrence or distant metastasis are not rare in low-grade tumors. Accordingly, it is advised that adjuvant therapy be performed after hysterectomy to prevent recurrence or distant metastasis.

\section{REFERENCES}

1. Echt G, Jepson J, Steel J, Langholz B, Luxton G, Hernandez W, et al. Treatment of uterine sarcomas. Cancer. 1990;66:35-9.

2. Bodner K, Bodner-Adler B, Obermair A, Windbichler G, Petru E, Mayerhofer S, et al. Prognostic parameters in endometrial stromal sarcoma: a clinicopathologic study in 31 patients. Gynecol Oncol. 2001;81:160-5.

3. Gadducci A, Sartori E, Landoni F, Zola P, Maggino T, Urgesi A, et al. Endometrial stromal sarcoma: analysis of treatment failures and survival. Gynecol Oncol. 1996;63:247-53.

4. Norris HJ, Taylor HB. Mesenchymal tumors of the uterus. I. A clinical and pathological study of 53 endometrial stromal tumors. Cancer. 1966;19:755-66.

5. Amant F, Vergote I, Moerman P. The classification of a uterine sarcoma as 'high-grade endometrial stromal sarcoma' should be abandoned. Gynecol Oncol. 2004;95:412-3.

6. Chang KL, Crabtree GS, Lim-Tan SK, Kempson RL, Hendrickson MR. Primary uterine endometrial stromal neoplasms. A clinicopathologic study of 117 cases. Am J Surg Pathol. 1990;14:415-38.

7. Li AJ, Giuntoli RL 2nd, Drake R, Byun SY, Rojas F, Barbuto $\mathrm{D}$, et al. Ovarian preservation in stage I low-grade endometrial stromal sarcomas. Obstet Gynecol. 2005;106:1304-8.

8. Berchuck A, Rubin SC, Hoskins WJ, Saigo PE, Pierce VK, Lewis JL. Treatment of endometrial stromal tumors. Gynecol Oncol. 1990;36:60-5.

9. Mansi JL, Ramachandra S, Wiltshaw E, Fisher C. Endometrial stromal sarcomas. Gynecol Oncol. 1990;36:113-8.

10. Creasman WT, Morrow CP, Bundy BN, Homesley HD, Graham JE, Heller PB. Surgical pathologic spread patterns of endometrial cancer. A Gynecologic Oncology Group Study. Cancer. 1987;60:2035-41.

11. Bohr L, Thomsen CF. Low-grade stromal sarcoma: a benign appearing malignant uterine tumour; a review of current literature. Differential diagnostic problems illustrated by four cases. Eur J Obstet Gynecol Reprod Biol. 1991;39:63-9.

12. Cacciatore B, Lehtovirta P, Wahlstrom T, Ylostalo P. Ultra- sound findings in uterine mixed mullerian sarcomas and endometrial stromal sarcomas. Gynecol Oncol. 1989;35:290-3.

13. Ueda M, Otsuka M, Hatakenaka M, Sakai S, Ono M, Yoshimitsu $\mathrm{K}$, et al. MR imaging findings of uterine endometrial stromal sarcoma: differentiation from endometrial carcinoma. Eur Radiol. 2001;11:28-33.

14. Leath CA 3rd, Huh WK, Hyde J Jr, Cohn DE, Resnick KE, Taylor NP, et al. A multi-institutional review of outcomes of endometrial stromal sarcoma. Gynecol Oncol. 2007;105:630-4.

15. Lin YC, Kudelka AP, Tresukosol D, Malpica A, Carrasco CH, Lawrence DD, et al. Prolonged stabilization of progressive endometrial stromal sarcoma with prolonged oral etoposide therapy. Gynecol Oncol. 1995;58:262-5.

16. Riopel J, Plante M, Renaud MC, Roy M, Tetu B. Lymph node metastases in low-grade endometrial stromal sarcoma. Gynecol Oncol. 2005;96:402-6.

17. Haberal A, Kayikcioglu F, Boran N, Caliskan E, Ozgul N, Kose MF. Endometrial stromal sarcoma of the uterus: analysis of 25 patients. Eur J Obstet Gynecol Reprod Biol. 2003;109: 209-13.

18. Sutton G, Blessing JA, Park R, DiSaia PJ, Rosenshein N. Ifosfamide treatment of recurrent or metastatic endometrial stromal sarcomas previously unexposed to chemotherapy: a study of the Gynecologic Oncology Group. Obstet Gynecol. 1996;87:747-50.

19. Muss HB, Bundy B, DiSaia PJ, Homesley HD, Fowler WC $\mathrm{Jr}$, Creasman W, et al. Treatment of recurrent or advanced uterine sarcoma. A randomized trial of doxorubicin versus doxorubicin and cyclophosphamide (a phase III trial of the Gynecologic Oncology Group). Cancer. 1985;55:1648-53.

20. Szlosarek PW, Lofts FJ, Pettengell R, Carter P, Young M, Harmer C. Effective treatment of a patient with a high-grade endometrial stromal sarcoma with an accelerated regimen of carboplatin and paclitaxel. Anticancer Drugs. 2000;11:275-8.

21. Yamawaki T, Shimizu Y, Hasumi K. Treatment of stage IV "high-grade" endometrial stromal sarcoma with ifosfamide, adriamycin, and cisplatin. Gynecol Oncol. 1997;64:265-9.

22. Chu MC, Mor G, Lim C, Zheng W, Parkash V, Schwartz PE. Low-grade endometrial stromal sarcoma: hormonal aspects. Gynecol Oncol. 2003;90:170-6.

23. Pink D, Lindner T, Mrozek A, Kretzschmar A, Thuss-Patience PC, Dorken B, et al. Harm or benefit of hormonal treatment in metastatic low-grade endometrial stromal sarcoma: single center experience with 10 cases and review of the literature. Gynecol Oncol. 2006;101:464-9.

24. Amant F, De Knijf A, Van Calster B, Leunen K, Neven P, Berteloot $\mathrm{P}$, et al. Clinical study investigating the role of lymphadenectomy, surgical castration and adjuvant hormonal treatment in endometrial stromal sarcoma. Br J Cancer. 2007; 97:1194-9.

25. Weitmann HD, Knocke TH, Kucera H, Potter R. Radiation therapy in the treatment of endometrial stromal sarcoma. Int J Radiat Oncol Biol Phys. 2001;49:739-48. 